\title{
Peripheral IGF-1 in bipolar disorder and major depressive disorder: a systematic review and meta-analysis
}

\author{
Mengyi Chen^, Lei Zhang^, Qi Jiang^ \\ Department of Geriatric, Shanghai Pudong New District Mental Health Center, Shanghai, China \\ Contributions: (I) Conception and design: M Chen; (II) Administrative support: M Chen; (III) Provision of study materials or patients: L Zhang, Q \\ Jiang; (IV) Collection and assembly of data: L Zhang, Q Jiang; (V) Data analysis and interpretation: M Chen; (VI) Manuscript writing: All authors; (VII) \\ Final approval of manuscript: All authors. \\ Correspondence to: Mengyi Chen. Shanghai Pudong New District Mental Health Center, Shanghai 200124, China. Email: 1141134986@qq.com.
}

\begin{abstract}
Backgrounda In recent years, a number of studies have shown abnormal levels of peripheral insulin growth factor-1 (IGF-1) in patients with mood disorder, but the results are not uniform. Therefore, this paper focuses on such studies, conducts a systematic review and meta-analysis, and discusses the factors affecting peripheral IGF-1 in patients with mood disorder.

Methods: Cochrane database, PubMed database, Embase database, CNKI database, Wanfang Database and Weipu database were searched by computer. The retrieval time was from June 2020 to search for a controlled study of the relationship between bipolar disorder (BD) or major depressive disorder and normal control peripheral IGF-1. Review Manager (version 5.3) software was used for meta-analysis.

Results: A total of 14 articles in Chinese and English were included; 285 patients with BD and 503 patients with major depressive disorder. Meta-analysis showed that in comparison with the control group, IGF1 levels in peripheral blood of patients with $\mathrm{BD}(\mathrm{MD}=67.66,95 \% \mathrm{CI}: 7.01-128.31, \mathrm{P}=0.03)$ and major depressive disorder ( $\mathrm{MD}=8.01,95 \% \mathrm{CI}: 3.43-12.58, \mathrm{P}=0.0006)$ were significantly increased. In the metaanalysis comparing the peripheral IGF-1 levels of patients before and after treatment, the results showed no significant change in the peripheral IGF-1 level before and after treatment $(\mathrm{P}=0.53)$.
\end{abstract}

Conclusions: High peripheral IGF-1 level is a related factor of BD and major depressive disorder, although this needs to be confirmed by further large sample studies.

Keywords: Meta-analysis; bipolar disorder (BD); major depressive disorder; IGF-1.

Submitted Sep 09, 2020. Accepted for publication Nov 06, 2020.

doi: 10.21037/apm-20-1967

View this article at: http://dx.doi.org/10.21037/apm-20-1967

\section{Introduction}

Insulin-like growth factor (IGF) is a type of broadspectrum growth-promoting factor. Its chemical structure is similar to proinsulin and is homologous to a single chain polypeptide. IGF combines with insulin-like growth factor binding protein (IGFBP) in tissues or blood to play its physiological roles (1) which include major functions in the growth and development of human tissues and cells.
The synthesis and secretion of IGF is controlled by the level of growth hormone $(\mathrm{GH})$ in the blood and IGF has a negative feedback regulating effect on the secretion of GH in circulation, thus forming the GH/IGF axis. The most widely studied IGF is IGF-1. IGF-1 is a polypeptide chain consisting of 70 amino acids. Eighty percent of the IGF-1 in human peripheral blood is synthesized and secreted by the liver (2,3), but the expression of IGF-1 is distributed in almost all tissues (4). IGF-1 has a wide range of biological

^ ORCID: Mengyi Chen, 0000-0002-9971-0787; Lei Zhang, 0000-0003-2404-7471; Qi Jiang, 0000-0001-8185-5876. 
functions, which include the promotion of glucose metabolism and sugar transport, the acceleration of fat and glycogen synthesis, facilitating DNA, RNA and protein synthesis, motivating cell proliferation and differentiation, inhibiting cell apoptosis and regulating the immune process.

IGF-1 also plays an important role in the central nervous system. Studies have shown that IGF-1 can affect the structure and function of synapses $(5,6)$, regulate the glucose metabolism of brain cells (7), inhibit the apoptosis of neurons and glial cells $(8,9)$, regulate enzyme activity $(6,10)$, reduce the damage caused by various pathological factors to the central nervous system $(11,12)$, and play an important protective role in the growth, development and remodeling of nerve tissues. In recent years, there have been continuous studies on IGF-1 in psychiatric patients. In 2016, Tu et al. (13) conducted a meta-analysis of six studies on IGF-1 levels in the peripheral blood of patients with major depressive disorder (MDD) and three studies on IGF-1 levels in the peripheral blood of patients with bipolar disorder (BD). Similar studies in recent years have found something different. According to a case control study from Italy, there was no statistically significant difference in peripheral IGF-1 level between MDD patients and healthy controls [25], while a study from China in the same year showed that the peripheral IGF-1 level of MDD patients was significantly higher than that of healthy controls [27]. Therefore, we summarized 14 studies on peripheral blood IGF-1 levels of patients with mood disorders from 1988 to 2020, where five were related to BD (14-18) and nine were related to MDD (19-27). A literature review and metaanalysis were performed here. We present the following article in accordance with the PRISMA reporting checklist (available at http://dx.doi.org/10.21037/apm-20-1967).

\section{Methods}

\section{Literature search}

The following databases were queried; Cochrane database, PubMed database, Embase database, Chinese Journal FullText Database (CNKI), Wanfang Database and Weipu database. The retrieval time was set up until June 2020 . The following English retrieval keywords and titles were used to search ("IGF-1" OR "insulin-like growth factor 1") AND ("depression" OR "bipolar disorder" OR "mood disorder" OR "mental disorder" OR "depressive disorder"
OR “affective disorder” OR “mania”).

\section{Inclusion and exclusion criteria}

Inclusion criteria: (I) published studies in English and Chinese on the relationship between BD, MDD and peripheral blood IGF-1; (II) the subjects were diagnosed as BD or MDD according to DSM or ICD standards; (III) complete data to carry out the original analysis; (IV) have at least one set of control study. Exclusion criteria: (I) review, case reports and other non-academic literature and non-clinical studies; (II) The samples were not from the peripheral blood; (III) the datasets were incomplete and the original IGF-1 values of peripheral blood could not be extracted; (IV) lack of normal control group. Data extraction and risk assessment of literature: literature search, data extraction and quality assessment were conducted independently by two doctors. If the two doctors disagreed, the third doctor would assess the results. The screening and search protocols are shown in Figure 1.

\section{Statistical analysis}

To control for confounding factors and to reduce heterogeneity, we extracted from the original literature the age, sex, race, sample source (peripheral blood or serum or plasma), sample extraction time, medication situation, detection mode (ELISA or RIA), and performed subgroup analysis based on the above factors. In order to exclude the possible confounding effects of drugs, we conducted an additional meta-analysis to explore the effect of treatment on peripheral IGF-1 levels in four of the studies that recorded both pre-treatment and post-treatment IGF-1 levels.

Meta-analysis of data was performed using Revman software (version 5.3). Mean difference (MD) and $95 \%$ confidence intervals (95\% CI) were used for IGF-1 levels. The heterogeneity of the study was tested by the heterotopic coefficient $\mathrm{I}^{2}$. Heterogeneity between studies was determined by $\mathrm{P}$ value and $\mathrm{I}^{2}$ test where $\mathrm{P}>0.05$ and $\mathrm{I}^{2}<50 \%$ indicated no statistical heterogeneity between studies. A fixed effect model was used for the meta-analysis. If $\mathrm{P} \leq 0.05$ or $\mathrm{I}^{2} \geq 50 \%$, there was statistical heterogeneity among studies. A random effect model was used for metaanalysis. The source of heterogeneity and its influence on the stability of results were found by sensitivity analysis. 


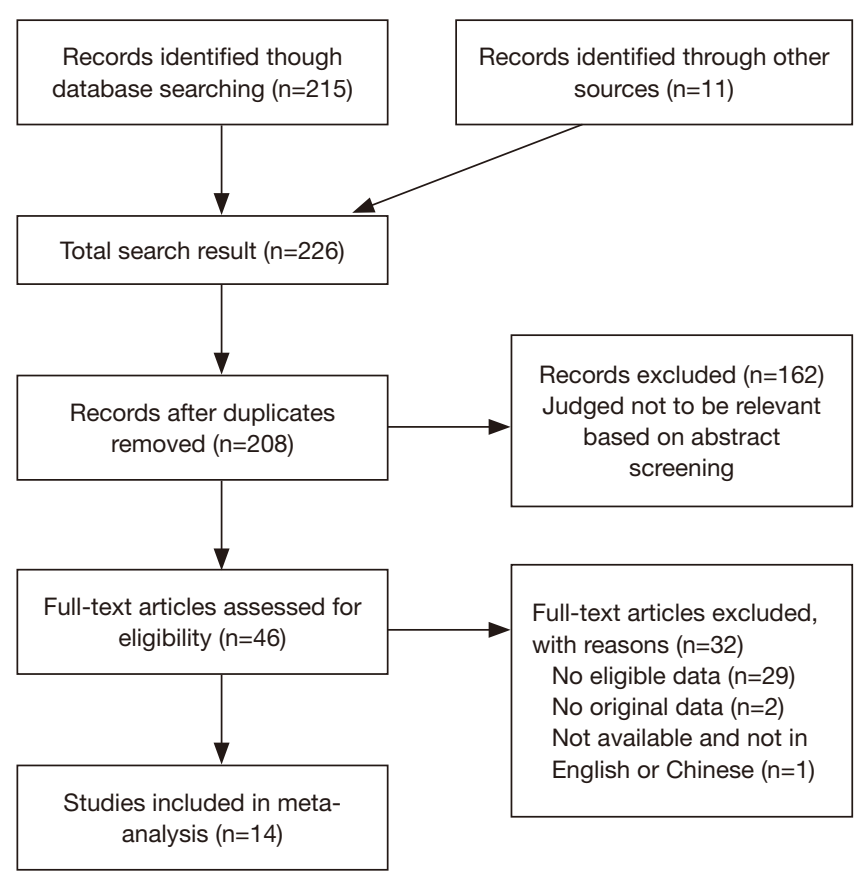

Figure 1 Study identification and literature review process.

$\mathrm{P} \leq 0.05$ was considered statistically significant. Funnel plots were used to assess publication bias.

\section{Results}

\section{Studies included in each meta-analysis}

A total of 237 related studies were searched for in the included study against the English database and the Chinese database, among which 211 were in English and 26 were in Chinese. After reading the title and abstract, 187 were excluded and 36 were excluded after reading the full text. Finally 14 (14-27) were confirmed to be included in the meta-analysis which included 12 English studies (14-25) and two Chinese studies $(26,27)$. The data of the selected studies are shown in Table 1.

\section{The main results of the current meta-analysis}

A total of 14 studies were included, nine of which focused on MDD (19-27). A total of 503 patients and 488 controls were included. Five studies on BD (14-18) included 285 patients and 274 controls. A random effect model was used for meta-analysis. The meta-analysis revealed that the peripheral IGF-1 level of BD and MDD patients was significantly higher than that of normal controls
$(\mathrm{MD}=11.23,95 \%$ CI: 6.29-16.18, $\mathrm{P}<0.00001)$. When BD $(\mathrm{MD}=67.66,95 \% \mathrm{CI}: 7.02-128.31, \mathrm{P}=0.03)$ and $\mathrm{MDD}$ ( $\mathrm{MD}=8.01,95 \% \mathrm{CI}: 3.43-12.58, \mathrm{P}=0.0006)$ were compared separately the peripheral IGF-1 levels were significantly increased in both groups (Figure 2). The heterogeneity test indicated that there was heterogeneity in the included articles $\left(\mathrm{I}^{2}=95 \%\right.$ in $\mathrm{BD}+\mathrm{MDD}, \mathrm{I}^{2}=91 \%$ in $\mathrm{BD}$ group, $\mathrm{I}^{2}=96 \%$ in MDD group), and that subgroup analysis was required to explore the source of heterogeneity.

\section{Investigation of heterogeneity and bias}

Funnel plots of the 14 studies included in this meta-analysis are shown in Figure 3. The scatter points of validity of each study are arranged symmetrically around the centerline in an inverted funnel shape which indicates that the literature in this meta-analysis had little publication bias.

\section{Subgroup meta-analysis}

The main results of subgroup meta-analysis of studies which included the medication situation

The studies can be divided according to whether patients are drug naive or drug free, which includes the Yes group $(14-16,19,21-23,25,26)$ and the No group $(17,18,20,24,27)$. This showed that the medication situation is a source of heterogeneity as peripheral levels of IGF-1 were higher in both subgroups compared to normal control groups.

\section{The main results of subgroup meta-analysis of studies with sample source}

According to the sample source, the included studies can be divided into three subgroups; plasma subgroup $(15,19,21)$, serum subgroup (16-18,20,22-27) and peripheral blood subgroup (14). These results show that sample source is a source of heterogeneity as peripheral levels of IGF-1 were higher in the peripheral blood subgroups and serum subgroup than in the normal control group.

\section{The main results of subgroup meta-analysis of studies with different methods of measuring}

According to the different methods of measuring, the included studies can be divided into three subgroups; ELISA subgroup (14-16,18,24,25,27), RIA subgroup $(19-21,23,26)$ and CL subgroup (17). The results reveal that different methods of measuring is a source of heterogeneity 


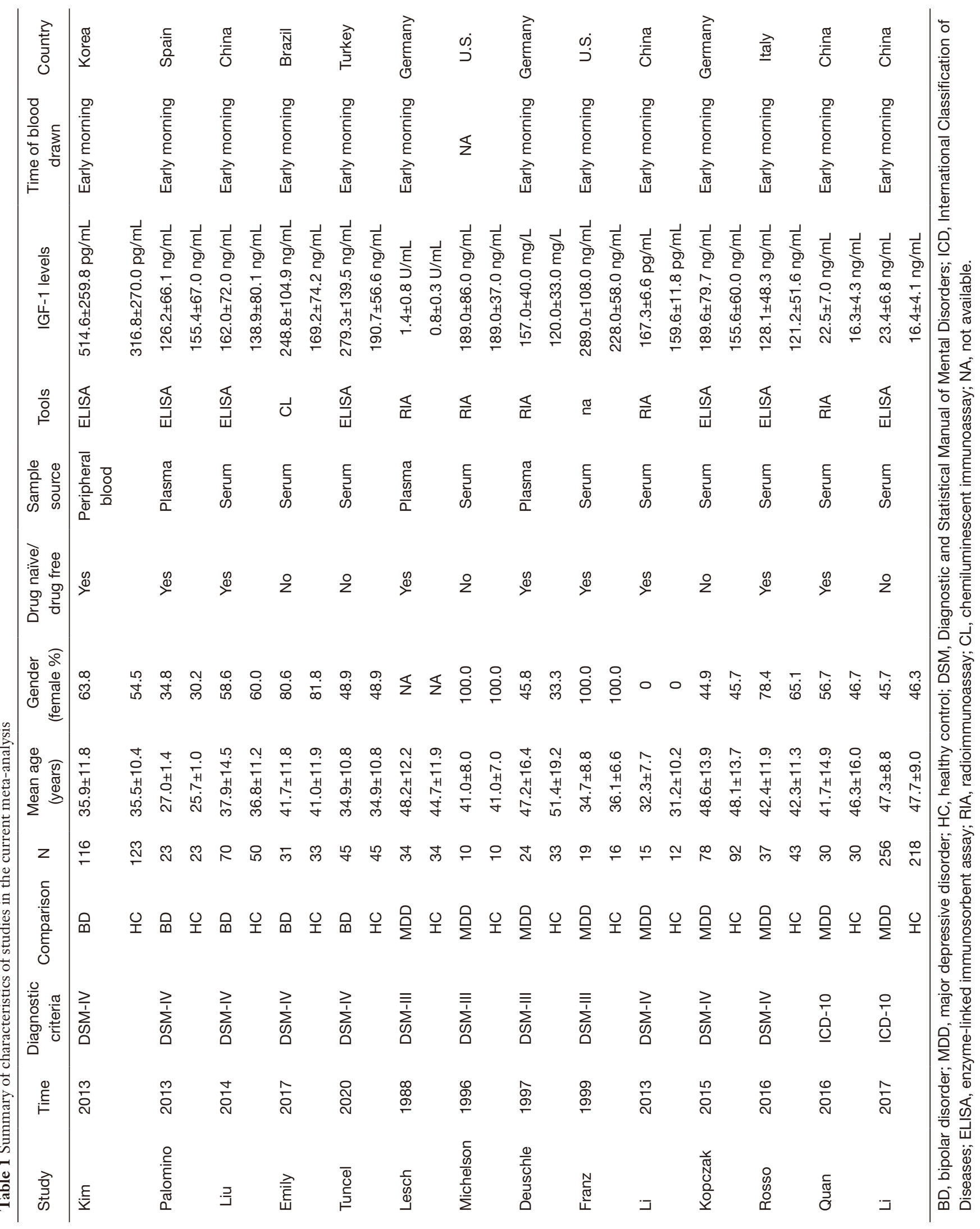




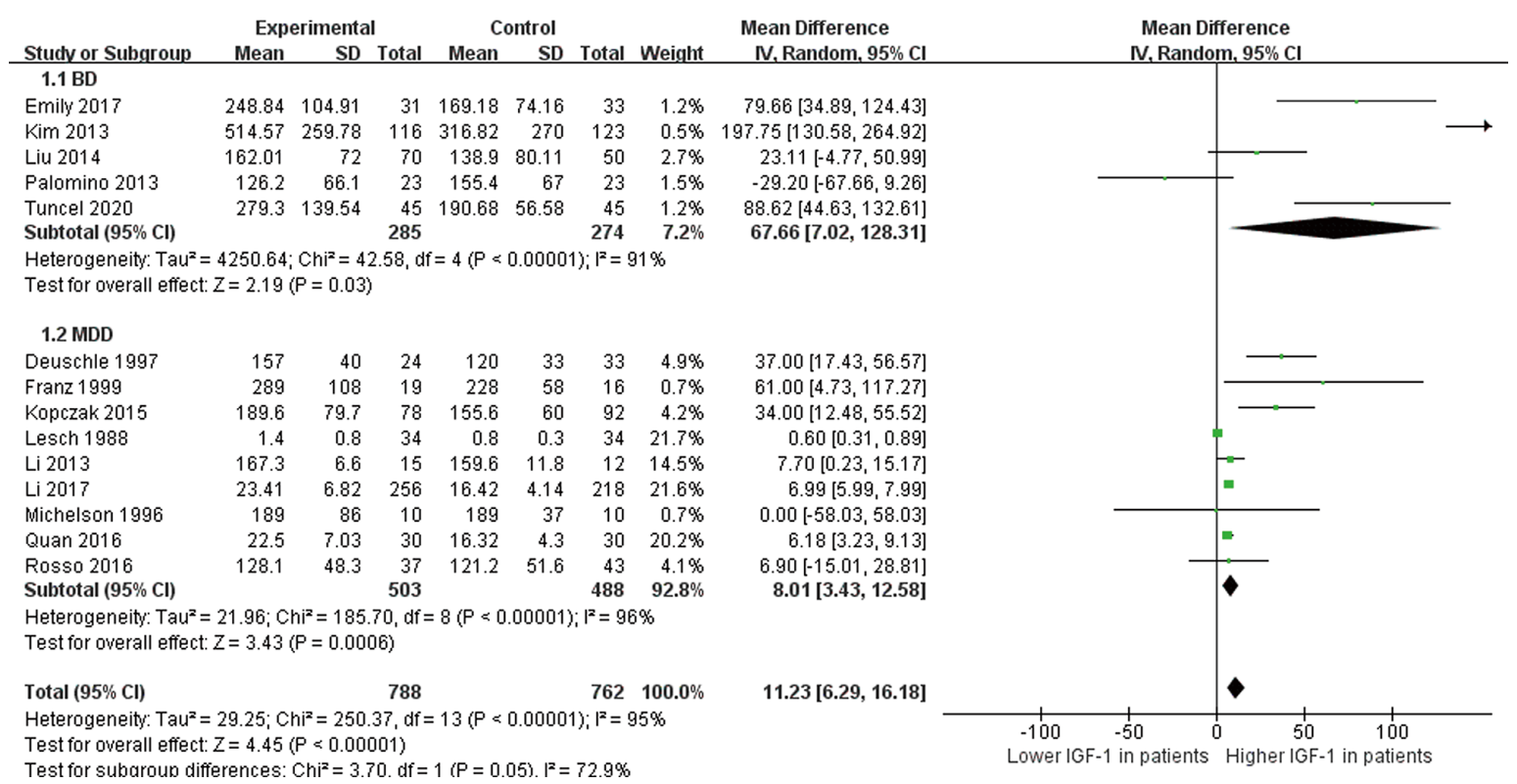

Figure 2 Meta-analysis of peripheral IGF-1 in bipolar disorder and major depressive disorder. SD, standard deviation; IV, inverse variance; 95\% CI, 95\% confidence interval; IGF-1, Insulin-like growth factor 1.

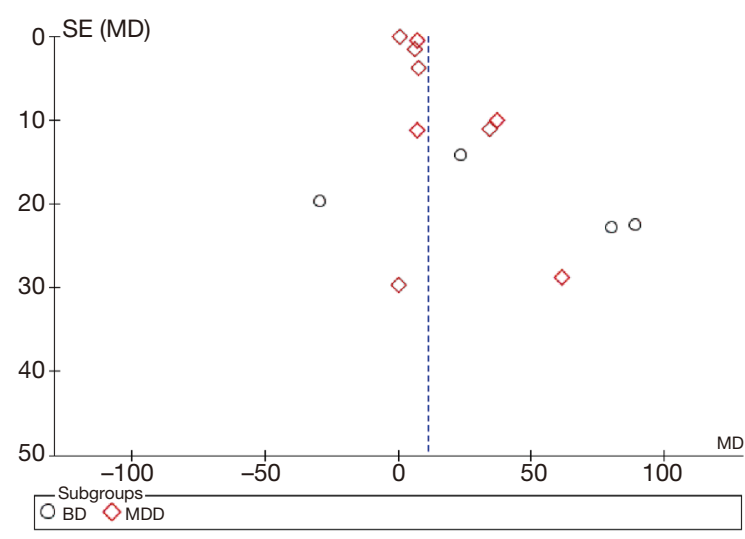

Figure 3 The funnel plot of meta-analysis. BD, bipolar disorder; MDD, major depressive disorder.

as peripheral level of IGF-1 was higher in all three subgroups compared to the normal control group.

\section{The main results of subgroup meta-analysis of studies with the time of blood drawn}

When considering that the level of peripheral blood IGF-1 is affected by food intake, the time of blood drawn may be a source of heterogeneity. According to the time of blood drawn, and excluding the study that did not specify the time of blood collection (20), the studies can be divided into two subgroups; early morning subgroup (14-19,21-22,24-27) and not early morning subgroup (23). The results showed that the time of blood drawn was not the source of heterogeneity. However, whether the time is in the morning or not, it was correlated with peripheral IGF-1 levels, and peripheral IGF-1 levels in both subgroups were higher than those in the normal control group.

\section{The main results of subgroup meta-analysis of studies with the participants race}

According to the participants race, the included studies can be divided into three subgroups; Asia subgroup $(14,16,18,23,26,27)$, American subgroup $(17,20,22)$ and Europe subgroup $(15,19,21,24,25)$. These result shows that the participants race is a source of heterogeneity, and peripheral level of IGF-1 was higher in both Asia subgroup and American subgroup than in the normal control group.

All subgroup analysis results are shown in Table 2.

\section{The main results of meta-analysis of before and after treatment}

In the 14 included studies some could not exclude the influence of psychiatric drugs on peripheral blood IGF- 
Table 2 Subgroup analysis of periphery IGF-1 level of BD and MDD patients

\begin{tabular}{|c|c|c|c|c|c|c|c|}
\hline Subgroup & & \multicolumn{2}{|c|}{ Heterogeneity test } & MD & $95 \% \mathrm{Cl}$ & Z & $\mathrm{P}$ \\
\hline \multirow[t]{2}{*}{ Drug naïve/drug free } & Yes & $<0.00001$ & 87 & 9.08 & $2.04-16.12$ & 2.53 & 0.01 \\
\hline & No & $<0.00001$ & 86 & 39.54 & $8.10-70.97$ & 2.47 & 0.01 \\
\hline \multirow[t]{2}{*}{ Sample source } & Plasma & 0.0004 & 87 & 6.10 & -23.10 to 35.29 & 0.41 & 0.68 \\
\hline & Peripheral blood & - & - & 197.75 & $89.60-305.90$ & 3.58 & 0.0003 \\
\hline \multirow[t]{3}{*}{ Methods of measuring } & ELISA & $<0.00001$ & 83 & 25.43 & $3.72-47.13$ & 2.30 & 0.02 \\
\hline & $\mathrm{RIA}$ & $<0.00001$ & 87 & 6.71 & $0.79-12.63$ & 2.22 & 0.03 \\
\hline & $\mathrm{Cl}$ & - & - & 79.66 & $34.89-124.43$ & 3.49 & 0.0005 \\
\hline \multirow[t]{3}{*}{ Participants race } & Asian & 0.0001 & 80 & 9.78 & $4.22-15.34$ & 3.45 & 0.0006 \\
\hline & America & 0.10 & 57 & 49.42 & $2.99-95.84$ & 2.09 & 0.04 \\
\hline & Europe & $<0.0001$ & 84 & 12.24 & -7.06 to 31.55 & 1.24 & 0.21 \\
\hline
\end{tabular}

IGF-1, Insulin-like growth factor 1; BD, bipolar disorder; MDD, major depressive disorder; ELISA, enzyme-linked immunosorbent assay; $\mathrm{RIA}$, radioimmunoassay; $\mathrm{Cl}$, chemiluminescent immunoassay.

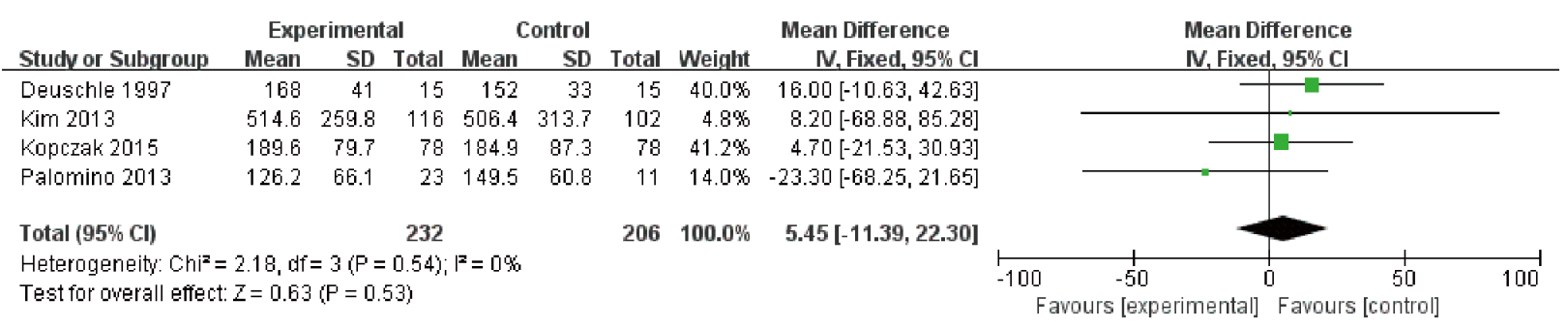

Figure 4 The meta-analysis of before and after treatment. SD, standard deviation; IV, inverse variance; $95 \%$ CI, $95 \%$ confidence interval.

1 levels. We then conducted another meta-analysis on the changes of peripheral blood IGF-1 levels before and after treatment. Five studies $(14,15,21,23,24)$ mentioned related content, among of which Kim (14) and Palomino (15) used mood stabilizers combined with antipsychotic drugs and Deuschle (21) and Kopczak (24) used antidepressant drugs. The above studies involved treatment for more than 24 days, and so blood was collected in the morning on an empty stomach, and so meta-analysis of drug influence was included. $\mathrm{Li}$ (23) used enalapril and compared the changes of IGF-1 in peripheral blood within 24 hours before and after the use of enalapril. These results were affected by circadian rhythm and eating situation, so this metaanalysis was not included. A fixed effect model was used for this meta-analysis. The results show that there were no significant change in IGF-1 level before and after treatment $\left(\mathrm{MD}=5.45,95 \% \mathrm{CI}:-11.39\right.$ to $\left.22.30, \mathrm{P}=0.53, \mathrm{I}^{2}=0 \%\right)$ (Figure 4).

\section{Discussion}

Previous studies have shown that the most important role of IGF-1 in the brain is to control cell growth, differentiation, tissue metabolism and promote DNA synthesis. However, a number of studies in recent years have shown that IGF-1, as a neurotrophic factor regulated by the immune system, can reduce the excitatory toxicity induced by aspartic acid (NMDA) and prevent neuronal autophagy (28). IGF-1 can 
also enhance the effects of anti-inflammatory cytokines IL-4 and IL-10, thereby negatively regulating inflammatory response in the brain. As the inflammation hypothesis of the central nervous system plays an important role in the mechanism of depression and $\mathrm{BD}$, we must consider special significance in the roles of IGF-1 in the field of mood disorders (29). Some researchers suggest that a decrease in IGF-1 expression in the brain disrupts neuroplasticity and promotes inflammatory pathways in the brain which leads to morphological deterioration in brain regions responsible for emotional and cognitive processes. From this perspective, the increased level of systemic IGF-1 observed in patients with mood disorder can be considered as a compensatory mechanism which enhances the activity of the hypothalamic-pituitary-growth promoting axis in response to insufficient cerebral IGF-1 concentration (30).

The results of this study show that peripheral IGF-1 levels of $\mathrm{BD}$ and MDD patients were significantly higher than that of the control group. These results were similar to a number of domestic and foreign studies (14,16-19,21-27), but inconsistent with the results of Palomino (15) and Michelson (20). This may be related to the sample size, disease course, patient age and other factors of the two studies. We found that peripheral IGF-1 levels were correlated with the sample source. The IGF-1 levels in the studies which used serum as the sample source were significantly higher than that in the control group, while IGF-1 levels in the study using plasma as the sample source were not significantly changed. According to the above 14 studies, most of the earlier studies used plasma as samples, but in the recent ten years, most studies chose serum as the sample source. Compared with plasma, serum can exclude the influence of fibrinogen and some coagulation factors, so it is more accurate.

Studies have shown that age, sex, body mass index (BMI), region, dietary structure and nutritional status are possible factors affecting the level of serum IGF-1. The level of serum IGF-1 in adults decreases with the increase of age, and the decline rate is first fast and then slow, and the decline rate of women is faster than that of men (31), such as the research results of Brabant et al. (32). There are gender differences in serum IGF-1 levels in adults, but the specific mechanism is not clear. The effect of gender on serum IGF1 may be mainly through estrogen. Studies have confirmed that estrogen has a clear inhibitory effect on the process of IGF-1 synthesis regulated by growth hormone (33). In order to exclude the heterogeneous effects of age and sex on serum IGF-1 levels, it is best to distinguish between age and sex grouping studies, and exclude oral contraceptive and other factors that interfere with female growth hormone and hormone replacement therapy. Nutritional status and body composition affected the level of serum IGF-1, the level of growth hormone decreased and the level of serum IGF-1 increased in obese people (34), while in patients with malnutrition caused by cachexia or neurasthenia, the level of serum growth hormone increased and the level of IGF-1 decreased (35). In epidemiological studies, the relationship between serum IGF-1 level and BMI has not been determined. Physical exercise may also have an effect on serum IGF-1. In 1990, Kelly first reported in JCEM that the level of serum IGF-1 was related to fitness. People who exercise regularly have higher levels of serum IGF-1 (36). However, Porch et al. reported in 1997 that daily physical exercise could significantly reduce the content of body fat, but had no effect on the level of serum IGF-1 (37). This finding was especially confirmed in the elderly population $(38,39)$. We found that peripheral IGF-1 levels were correlated with the race of participants as patients in Asia and American have higher peripheral IGF-1 level than the normal control group, while patients in Europe were not as obvious. This may be related to different geographical and cultural diet habits. To investigate this further we are looking towards future larger sample sizes, especially from Africa and Oceania. Subgroup analysis revealed that heterogeneity was influenced by whether patients were drug naive or drug free and the detection method of IGF-1. However, the results of different subgroups show that the peripheral IGF-1 levels of patients with mood disorders were higher than that of the normal control group. Subsequent meta-analysis on the effect of treatment showed that peripheral IGF-1 levels do not change significantly with treatment, which reflects the specificity of IGF-1 as a biomarker for patients with mood disorders.

This meta-analysis has the following limitations; This study only showed that there was no significant difference in the age of BD and MDD patients between the control group and the control group, that is, age had no effect on the heterogeneity of included articles (MD $=0.02,95 \%$ CI: -0.08 to $\left.10.12, \mathrm{P}=0.64, \mathrm{I}^{2}=14 \%\right)$. However, all the literatures have not conducted a stratified study on patients of different ages. The average age among these studies were different, the probability of the metabolic syndrome was different in the different age subjects, and IGF-1 as a broadspectrum growth-promoting factor widely participate in the body's metabolism of sugar and fat transfer process, so there are many confounding factors which can affect peripheral IGF-1 level in patients. Secondly, there were differences 
in the degree of disease onset in each study group. By onearm research and analysis, five studies (14-18) recorded the patient's Young Mania Rating Scale(YMRS) (ES $=25.349$, $95 \%$ CI: 7.441 to $43.257, \mathrm{P}=0.006, \mathrm{I}^{2}=99.8 \%$ ), and in the terms of nine studies on MDD, the Hamilton Depression Rating Scale (HAMD) was recorded in six studies $(17,19,21,22,25,26)(\mathrm{ES}=23.196,95 \%$ CI: 16.846 to 29.545 , $\left.\mathrm{P}<0.001, \mathrm{I}^{2}=98.9 \%\right)$. In the terms of the five studies on BD, three were during a manic episode, one was in remission and one was not indicated. Thus it can be seen that there is a great heterogeneity among the evaluation results of the disease degree of each study. The lack of unified evaluation criteria and grouping of evaluation results are common problems. Studies have shown that the severity of mood disorders can affect serum IGF-1 levels (40), but in this meta-analysis, due to the lack of relevant information, we are unable to move from qualitative to quantitative data. Finally, there are potential confounding factors which influence the reliability of the results, such as the fact that diagnostic criteria for diseases have been updated several times over the years.

IGF-1, as a polypeptide widely existing in various tissues and organs of human body, is involved in and regulates multiple physiological activities, and its special significance in the field of psychiatry is being explored. In recent years, researchers have found abnormalities in IGF-1 levels in Alzheimer's disease, obsessive-compulsive disorder, alcohol dependence, eating disorders and other psychological diseases (25,41-43). Animal experiments show that IGF-1 gene knockouts reduce a mice's ability to adapt to the environment and the degree of task completion decreases obviously. A subsequent study found that exogenous IGF-1 can reverse these abnormal behavior and ability to adapt (44). According to the above study, researchers presented the hypothesis that IGF-1 gene knockout mice can function as animal model of depression, and that IGF-1 has potential antidepressant effect (45). Zhang et al. used chronic unpredictable mild stress (CUMS) to established depression rat model and injected $5 \mathrm{~mL}$ IGF-1 $(5 \mathrm{mg} / \mathrm{mL})$, the results suggest that IGF-1 may improve depression symptoms by mediating TDAG5 1 activation of PI3K/Akt/FoxO3a signal pathway in hippocampus of depression model rats (46). In the study of effective dose, Hiney et al. studied the effect of prepubertal IGF-1 on the release of luteinizing hormone-releasing hormone (LHRH) in the hypothalamus of female rats, and observed it with the minimum effective dose of $10 \mathrm{ng} / \mathrm{mL}$ and the maximum effective dose of $100 \mathrm{ng} / \mathrm{mL}$. The results showed that IGF-1 played a role in the median eminence, and the density of IGF-1 receptor was the highest in the median eminence of the brain, resulting in an increase in dose-dependent release of LHRH (47). However, the study does not involve the side effects of IGF-1, indicating that IGF-1 involves many aspects of neuroendocrine and its mechanism and side effects are still not clear. At the same time, it also reflects that the pathogenesis of depression involves many factors, which can be explained by the existing antidepressant therapy after a period of time. In the field of $\mathrm{BD}$, researchers found patients sensitive with lithium carbonate have high expression of IGF1 related genes (48). Additionally, it was observed in vitro that exogenous IGF-1 may induce the up-regulation of lithium carbonate response-related microRNAs (49). Therefore, IGF-1 may be involved in the pharmacological processing of lithium salt, and its gene expression level could provide a predictive role for the therapeutic effect of lithium salt. What specific role IGF-1 plays in the field of mood disorders, and how can we use its physiological and pharmacological effects benefit clinical patients, remains to be answered by future researchers.

\section{Conclusions}

In summary, IGF-1 in peripheral blood of patients with $\mathrm{BD}$ and major depressive disorder is higher than that of the normal population. IGF-1 can be used as a potential laboratory indicator for mood disorders.

\section{Acknowledgments}

Funding: None.

\section{Footnote}

Reporting Checklist: The authors have completed the PRISMA reporting checklist. Available at http://dx.doi. org/10.21037/apm-20-1967

Conflicts of Interest: All authors have completed the ICMJE uniform disclosure form (available at http://dx.doi. org/10.21037/apm-20-1967). The authors have no conflicts of interest to declare.

Ethical Statement: The authors are accountable for all aspects of the work in ensuring that questions related to the accuracy or integrity of any part of the work are appropriately investigated and resolved. 
Open Access Statement: This is an Open Access article distributed in accordance with the Creative Commons Attribution-NonCommercial-NoDerivs 4.0 International License (CC BY-NC-ND 4.0), which permits the noncommercial replication and distribution of the article with the strict proviso that no changes or edits are made and the original work is properly cited (including links to both the formal publication through the relevant DOI and the license). See: https://creativecommons.org/licenses/by-nc-nd/4.0/.

\section{References}

1. Guan J, Williams CE, Skinner SJ, et al. The effects of insulin-like growth factor (IGF)-1, IGF-2, and des-IGF-1 on neuronal loss after hypoxic-ischemic brain injury in adult rats: evidence for a role for IGF binding proteins. Endocrinology 1996;137:893-8.

2. Le Roith D. The insulin-like growth factor system. Exp Diabesity Res 2003;4:205-12.

3. Torres-Aleman I. Toward a comprehensive neurobiology of IGF-I. Dev Neurobiol 2010;70:384-96.

4. Cohick WS, Clemmons DR. The insulin-like growth factor. Annu Rev Physiol 1993;55:131-53.

5. Chik CL, Li B, Karpinski E, et al. Insulin and insulin-like growth factor-I inhibit the L-type calcium channel current in rat pinealocytes. Endocrinology 1997;138:2033-42.

6. Selinfreund RH, Blair LA. Insulin-like growth factor-1 induces a rapid increase in calcium currents and spontaneous membrane activity in clonal pituitary cells. Mol Pharmacol 1994;45:1215-20.

7. Cheng CM, Reinhardt RR, Lee WH, et al. Insulinlike growth factor 1 regulates developing brain glucose metabolism. Proc Natl Acad Sci USA 2000;97:10236-41.

8. Guan J, Belharz EJ, Skinner SJ, et al. Intracerebral transportation and cellular localisation of insulinlike growth factor- 1 following central administration to rats with hypoxic-ischemic brain injury. Brain Res 2000;853:163-73.

9. Johnston BM, Mallard EC, Williams CE, et al. Insulinlike growth factor- 1 is a potent neuronal rescue agent after hypoxic-ischemic injury in fetal lambs. J Clin Invest 1996;97:300-8.

10. Matilainen O, Arpalahti L, Rantanen V, et al. Insulin/ IGF-1 signal regulates proteasome activity through the deubiquitinating enzyme UBH-4. Cell Rep 2013;3:1980-95.

11. Gluckman PD, Guan J, Williams C, et al. Asphyxial brain injury-the role of the IGF system. Mol Cell Endocrinol 1998;140:95-9.
12. Trejo JL, Carro E, Garcia GE, et al. Role of insulin-like growth factor I signaling in neurodegenerative diseases. J Mol Med (Berl) 2004;82:156-62.

13. Tu KY, Wu MK, Chen YW, et al. Significantly higher peripheral insulin-like growth factor-1 levels in patients with major depressive disorder or bipolar disorder than in healthy controls. Medicine (Baltimore) 2016;95:e2411.

14. Kim YK, Na KS, Hwang JA, et al. High insulin-like growth factor-1 in patients with bipolar I disorder: a trait marker? J Affect Disord 2013;151:738-43.

15. Palomino A, Gonzalez PA, Martinez CM, et al. Relationship between negative symptoms and plasma levels of insulin-like growth factor 1 in first-episode schizophrenia and bipolar disorder patients. Prog Neuropsychopharmacol Biol Psychiatry 2013;44:29-33.

16. Liu X, Zhang T, He S, et al. Elevated serum levels of FGF-2, NGF and IGF-1 in patients with manic episode of bipolar disorder. Psychiatry Res 2014;218:54-60.

17. da Silva EG, Pfaffenseller B, Walz J, et al. Peripheral insulin-like growth factor 1 in bipolar disorder. Psychiatry Res 2017;250:30-4.

18. Tunçel ÖK, Sarisoy G, Cetin E, et al. Neurotrophic factors in bipolar disorders patients with manic episode. Turk J Med Sci 2020;50:985-93.

19. Lesch KP, Rupprecht R, Muller U, et al. Insulin-like growth factor Iin depressed patients and controls. Acta Psychiatr Scand 1988;78:684-8.

20. Michelson D, Stratakis C, Hill L, et al. Bone mineral density in women with depression. N Engl J Med 1996;335:1176-81.

21. Deuschle M, Blum WF, Strasburger CJ, et al. Insulin-like growth factor-I (IGF-I) plasma concentrations are increased in depressed patients. Psychoneuroendocrinology 1997;22:493-503.

22. Franz B, Buysse DJ, Cherry CR, et al. Insulin-like growth factor 1and growth hormone binding protein in depression: a preliminary communication. J Psychiatr Res 1999;33:121-7.

23. Li SX, Liu LJ, Xu LZ, et al. Diurnal alterations in circadian genes and peptides in major depressive disorder before and after escitalopram treatment. Psychoneuroendocrinology 2013;38:2789-99.

24. Kopczak A, Stalla GK, Uhr M, et al. IGF-I in major depression and antidepressant treatment response. Eur Neuropsychopharmacol 2015;25:864-72.

25. Rosso G, Zanardini R, Chiodelli DF, et al. Serum level of insulin-like growth factor-1 and obsessive-compulsive disorder: a case-control study. Neuropsychobiology 2016;74:15-21. 
26. Quan JJ, Yang Z. Correlation between serum insulinlike growth factor (IGF-1) concentration and depression. Gansu Science and Technology 2016;32:133-5.

27. Li Q1, Guo Q. The application value of serum IGF-1, AVP, BDNF level combined detection in the assessment of depressive patients. Journal of Medical Forum 2017;38:143-5.

28. Li Y, Sun W, Han S, et al. IGF-1-Involved Negative Feedback of NR2B NMDA Subunits Protects Cultured Hippocampal Neurons Against NMDA-Induced Excitotoxicity. Mol Neurobiol 2017;54:684-96.

29. Szczęsny E, Slusarczyk J, Głombik K, et al. Possible contribution of IGF-1 to depressive disorder. Pharmacol Rep 2013;65:1622-31.

30. Levada OA, Troyan AS. Major depressive disorder and accelerated aging from a peripheral IGF-1 overexpression perspective. Med Hypotheses 2020;138:109610.

31. Kwan AY, Hartman ML. IGF-I measurements in the diagnosis of adult growth hormone deficiency. Pituitary 2007;10:151-7.

32. Brabant G, von zur Mühlen A, Wüster C, et al. Serum insulin-like growth factor I reference values for an automated chemiluminescence immunoassay system: results from a multicenter study. Horm Res 2003;60:53-60.

33. Ho K, Gibney J, Johannsson G, et al. Regulating of Growth Hormone Sensitivity by Sex Steroids: Implications for Therapy. Pituitary Today: Molecular, Physiological and Clinical Aspects, Basel: Karger Publishers, 2006;35:115-28.

34. Scacchi M, Pincelli AI, Cavagnini F. Growth hormone in obesity. Int J Obes Relat Metab Disord 1999;23:260-71.

35. Brabant G, Wallaschofski H. Normal levels of serum IGF-I: determinants and validity of current reference ranges. Pituitary 2007;10:129-33.

36. Kelly PJ, Eisman JA, Stuart MC, et al. Somatomedin-C, physical fitness, and bone density. J Clin Endocrinol Metab 1990;70:718-23.

37. Porch JV, Jain K, Reilly A, et al. Aging, physical activity, insulin-like growth factor I, and body composition in Guatemalan women. Am J Clin Nutr 1997;66:874-9.

38. Goodman-Gruen D, Barrett-Connor E. Epidemiology of insulin-like growth factor-I in elderly men and women. The Rancho Bernardo Study. Am J Epidemiol 1997;145:970-6.

39. Rudman D, Mattson DE. Serum insulin-like growth factor $\mathrm{I}$ in healthy older men in relation to physical activity. J Am Geriatr Soc 1994;42:71-6.

40. Ferensztajn-Rochowiak E, Kaczmarek M, Wójcicka M, et al. Glutamate-Related Antibodies and Peripheral Insulin-
Like Growth Factor in Bipolar Disorder and Lithium Prophylaxis. Neuropsychobiology 2019;77:49-56.

41. Calvo D, Gunstad J, Miller LA, et al. Higher serum insulin-like growth factor-1 is associated with better cognitive performance in persons with cognitive impairment. Psychogeriatrics 2013;13:170-4.

42. García-Marchena N, Silva-Peña D, Martín-Velasco AI, et al. Decreased plasma concentrations of BDNF and IGF1 in abstinent patients with alcohol use disorders. PLOS One 2017;12:e0187634.

43. Brambilla F, Santonastaso P, Caregaro L, et al. Growth hormone and insulin-like growth factor 1 secretions in eating disorders: Correlations with psychopathological aspects of the disorders. Psychiatry Res 2018;263:233-7.

44. Mitschelen M, Yan H, Farley JA, et al. Long-term deficiency of circulating and hippocampal insulin-like growth factor I induces depressive behavior in adult mice: a potential model of geriatric depression. Neuroscience 2011;185:50-60.

45. Mueller PL, Pritchett CE, Wiechman TN, et al. Antidepressant-like effects of insulin and IGF-1 are mediated by IGF-1 receptors in the brain. Brain Res Bull 2018;143:27-35.

46. Zhang X, Wang Y, Zhang J. Effect of IGF-1-mediated TDAG51 on hippocampal PI3K/Akt/FoxO3a signal pathway in depression model rats. Journal of Guangxi Medical University 2020;37:1228-35.

47. Hiney JK, Ojeda SR, Dees WL. Insulin-like growth factor I: a possible metabolic signal involved in the regulation of female puberty. Neuroendocrinology 1991;54:420-3.

48. Milanesi E, Hadar A, Maffioletti E, et al. Insulin-like Growth Factor 1 differentially Affects Lithium Sensitivity of Lymphoblastoid Cell Lines from Lithium Responder and Non-Responder Bipolar Disorder Patients. J Mol Neurosci 2015;56:681-7.

49. Squassina A, Costa M, Congiu D, et al. Insulin-like growth factor 1 (IGF-1) expression in up-regulated in lymphoblastoid cell lines of lithium responsive bipolar disorder patients. Pharmacol Res 2013;73:1-7.

(English Language Editor: L. Gray)

Cite this article as: Chen M, Zhang L, Jiang Q. Peripheral IGF-1 in bipolar disorder and major depressive disorder: a systematic review and meta-analysis. Ann Palliat Med 2020;9(6):4044-4053. doi: 10.21037/apm-20-1967 